\title{
Proteins Identified from Saliva and Salivary Glands of the Chinese Gall Aphid Schlechtendalia chinensis
}

\author{
Zixiang Yang, Lin Ma, Frederic Francis, Ying Yang, Hang Chen, Haixia Wu, \\ and Xiaoming Chen*
}

\begin{abstract}
Aphid saliva plays an essential role in the interaction between aphids and their host plants. Several aphid salivary proteins have been identified but none from galling aphids. Here the salivary proteins from the Chinese gall aphid are analyzed, Schlechtendalia chinensis, via an LC-MS/MS analysis. A total of 31 proteins are identified directly from saliva collected via an artificial diet, and 141 proteins are identified from extracts derived from dissected salivary glands. Among these identified proteins, 17 are found in both collected saliva and dissected salivary glands. In comparison with salivary proteins from ten other free-living Hemipterans, the most striking feature of the salivary protein from S. chinensis is the existence of high proportion of proteins with binding activity, including DNA-, protein-, ATP-, and iron-binding proteins. These proteins maybe involved in gall formation. These results provide a framework for future research to elucidate the molecular basis for gall induction by galling aphids.
\end{abstract}

feed on host plants. ${ }^{[4]}$ At present, little is known about the precise components in aphid saliva that play effector roles, especially for galling aphids. Here, the protein composition of $S$. chinensis saliva collected though a Parafilm diet sachet was analyzed and proteins from dissected salivary glands were also investigated based on LC-MS/MS analyses.

An S. chinensis aphid colony was maintained on $R$. chinensis and Plagiomnium maximoviczii hosts under natural conditions and wingless fundatrigeniae were obtained from fresh galls on $R$. chinensis trees. Saliva from wingless fundatrigeniae was collected following a modified protocol of Carolan et al. ${ }^{[5]}$ while salivary glands were obtained by dissecting wingless fundatrigeniae collected from fresh galls following

The Chinese gall aphid Schlechtendalia chinensis (Hemiptera, Aphididae, Eriosomatinae) induces galls on the Chinese sumac Rhus chinensis that are valuable for both industry and medicine due to the high level of gallotannins, which have been used in traditional Chinese medicine for more than 2000 years. ${ }^{[1]}$ Usually each gall is induced by a single aphid fundatrix and her offspring. Both gall initiation and growth manipulation in host plants are likely achieved through aphid salivary secretions, which are critical in an active interplay between host plants and gall aphids. ${ }^{[2]}$ Nevertheless, the functions of these aphid salivary proteins in the host plants remain unknown. Several lines of evidence suggested that aphid saliva is rich in secretory proteins that play roles analogous to effectors in plant-pathogen interactions. ${ }^{[3]}$ Effector proteins can elicit various physiological changes within host plants to create a favorable environment for insects and pathogens to

Dr. Z. Yang, L. Ma, Y. Yang, Dr. H. Cheng, Dr. H. Wu, Prof. X. Chen

Research Institute of Resource Insects

Chinese Academy of Forestry

Key Laboratory of Breeding and Utilization of Resource Insects of State

Forestry Administration

Kunming, Yunnan, P. R. China

E-mail: cafcxm@139.com

Dr. F. Francis

Functional and Evolutionary Entomology

Gembloux Agro-Bio Tech

Liege University

Gembloux, Belgium

DOI: 10.1002/pmic.201700378 a modified protocol of Mutti et al. ${ }^{[6]}$ One thousand wingless fundatrigeniae were collected from 24 replicates for saliva collection as presented in Figure 1. Multiple saliva collections were combined with equal volume of PBS, and then centrifuged twice through a 3000 molecular weight cutoff polyethersulfone membrane at $4{ }^{\circ} \mathrm{C}$. The extract was resuspended in $100 \mu \mathrm{L}$ of $9.5 \mathrm{~m}$ urea, 2\% CHAPS, $0.8 \%$ Pharmalyte $\mathrm{pH} 3-10$, and 1\% DTT. Salivary glands from wingless fundatrigeniae were dissected on sterile glass slides in ice-cold PBS using sterile fine needles. The glands were dissected and separated from the aphid bodies following the methods of Rao et al. ${ }^{[7]}$ Extracts of collected saliva and dissected salivary glands were combined with Laemmli buffer, separately. Proteins were separated on $13 \%$ resolving gels with a 4\% stacking gel prior to gel picture analysis using MagicScan 5.1 software (Suzhou, China).

After electrophoresis, visible protein bands were excised from preparative 1D gels destained, reduced, alkylated, and trypsindigested as described by Ben Amira et al. ${ }^{[8]}$ Generated peptides were analyzed by LC-MS/MS on a Q Exactive Mass Spectrometer (Thermo Fisher Scientific, UK). Peptides were separated using a BioBasic C18 at a flow rate of $200 \mathrm{~nL} \mathrm{m^{-1 }}$ and gradient of 5-95\% ACN over $45 \mathrm{~min}$. All data were acquired with the mass spectrometer operating in automatic data-dependent switching mode. A zoom scan was performed on the five most intense ions to determine charge state prior to an MS/MS analysis. Protein identification from the MS/MS data was performed using the TurboSEQUEST algorithm in BioWorks v. 3.2 to correlate the data against the pea aphid ACYPI proteins and UniProt databases. Precursor ion's mass tolerance was set at $\pm 0.1 \mathrm{Da}$, 


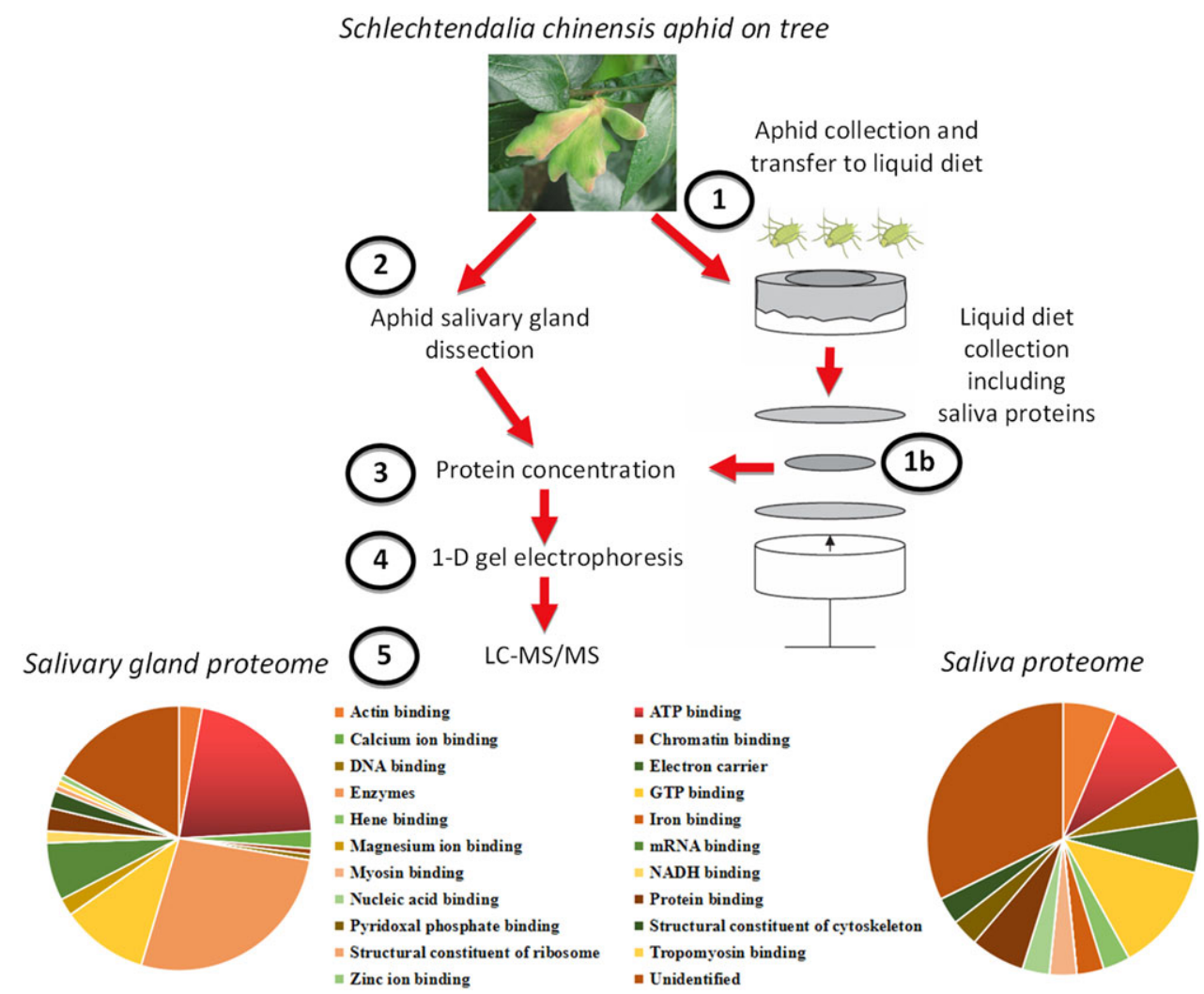

Figure 1. Experimental setup and protein functional distribution from saliva and salivary gland proteomes of the Chinese gall aphid.

the fragment ion's tolerance at \pm 0.2 Da with a maximum of two missed cleavage sites allowed. A transcriptomic database from the fundatrigenia of $S$. chinensis was also used for the protein searching and identification. Comparison between salivary proteins from $S$. chinensis and those from other insects was performed according to Huang et al. ${ }^{[9]}$ The mass spectrometry proteomics data have been deposited to the ProteomeXchange Consortium via the PRIDE ${ }^{[10]}$ partner repository with the dataset identifier PXD008928 and PXD008929.

A total of 31 saliva and 141 salivary gland proteins were identified after MASCOT searches of MS/MS spectra against UniProt (http://www.uniprot.org/) and an aphid database (https://bipaa.genouest.org/is/aphidbase/iagc/;Tables S1 and S2, Supporting Information). Among the 31 saliva and 141 salivary gland proteins, 20 and 64, respectively, were only detected in S. chinensis at present, and the remaining 11 and 77, respectively, have been reported in other free-living insects (Figure 2 and Table S3, Supporting Information). There were 17 proteins present in both saliva and dissected salivary glands of S. chinensis (Figure 2, Table 1 and Table S2, Supporting Information). Six of the these 17 proteins were novel, including a zinc finger protein, a cadherin, a piccolo isoform, a charybde-like isoform, and two unknown proteins (Table S3, Supporting Information). The remaining 11 proteins were also found in other free-living aphids. The same proteomic data were also searched against a $S$. chinensis transcriptome. However, no hits were found, which may be due to the low coverage of the transcriptome. Alternatively, no transcript hits were due to the fact that these two

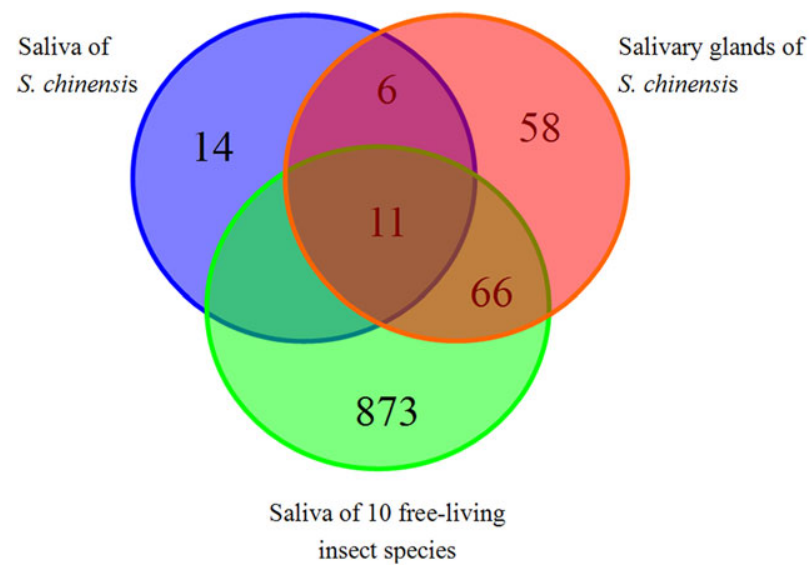

Figure 2. The Venn diagram of identified salivary proteins in the Chinese gall aphid and ten free-living insect species.

datasets were obtained from two different stages of the insect. Saliva and salivary glands were obtained from aphids collected from galls on the sumac, $R$. chinensis, whereas the transcriptome was generated from free-living aphids on the alternative host moss, P. maximoviczii.

One of the interesting characteristics of $S$. chinensis saliva and salivary gland proteins was the high proportion of proteins with various binding activities. For the 21 annotated proteins from saliva, 17 were with various binding functions 
Table 1. Comparative analysis of Chinese gall aphid salivary proteins with other insect species.

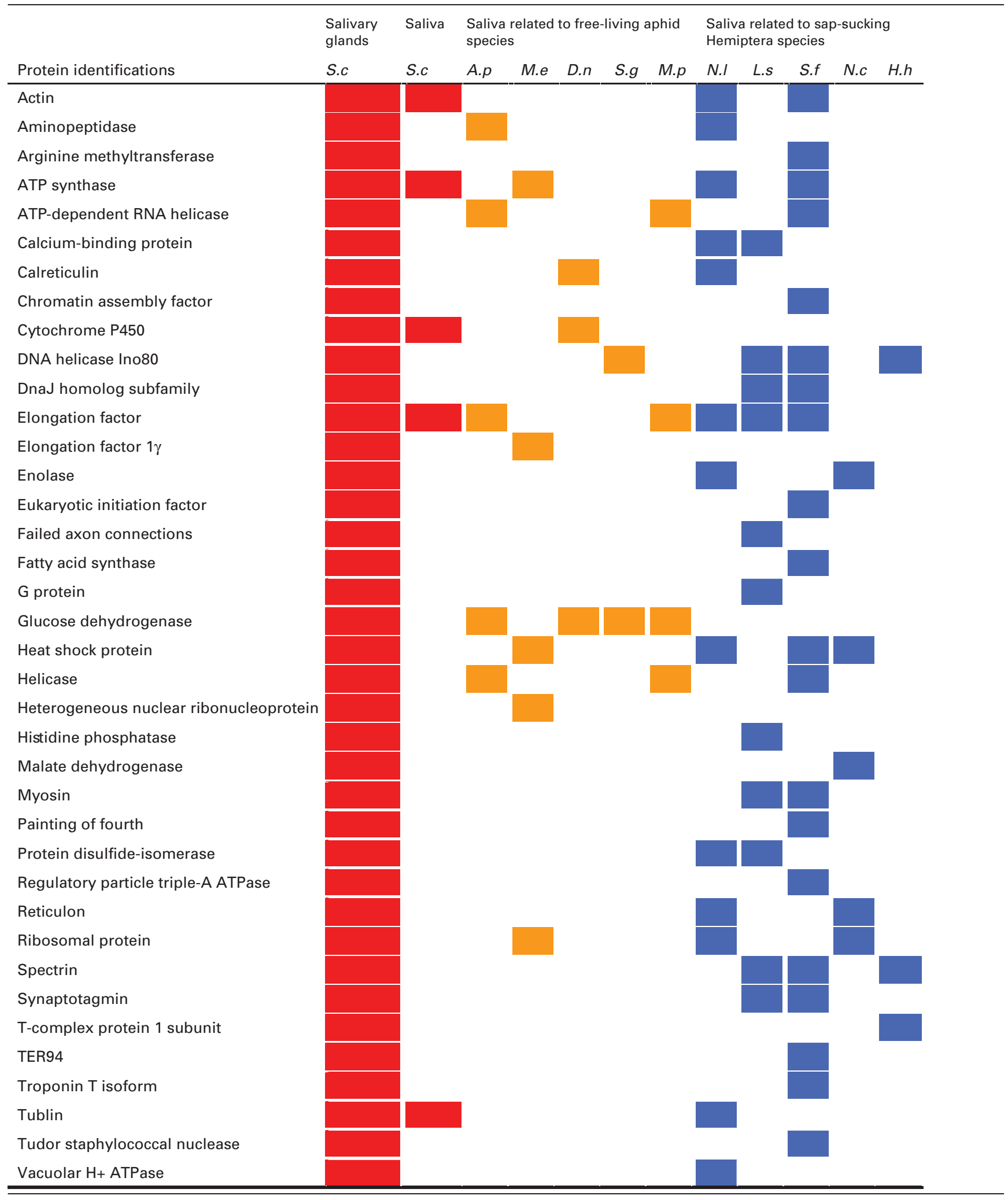

Red, orange, and blue areas are related to gall aphid, free-living aphids, and other free-living sucking Hemiptera species, respectively.; S.c, Schlechtendalia chinensis; A.p, Acyrthosiphon pisum; M.e, Macrosiphum euphorbiae; D.n, Diuraphis noxia; S.g, Schizaphis graminum; M.p, Myzus persicae; N.I, Nilaparvata lugens; L.s, Laodelphax striatellus; S.f, Sogatella furcifera; N.c, Nephotettix cincticeps; H.h, Halyomorpha halys. 
(Table S1, Supporting Information). For the 117 annotated proteins from dissected salivary glands, 75 were with various binding activities. The proteins with binding activities included ATP, GTP-, mRNA-, protein-, actin-, magnesium ion-, calcium ion-, NADH-, DNA-, chromatin-, tropomyosin-, and zinc ion-binding proteins. The second most abundant group of proteins was those with various enzymatic activities. Annotated enzymes included five dehydrogenases, four ATPases, three disulfide isomerases, three ubiquitin-protein transferases, two oxidoreductases, two endopeptidases, and one of each of the following: citrate synthase, aminobutyrate transaminase, and acid phosphatase. There were also three structural proteins and a translation elongation factor.

Aphid saliva plays multiple roles once injected into host tissues, including partial digestion of food prior to ingestion, detoxification, suppression of host defense, and serving as effectors for various host manipulations..$^{[2,11,12]}$ Unlike other free-living aphids, the feeding of a single $S$. chinensis aphid can induce the formation of a gall. It is very likely that some of aphid saliva proteins play a role in gall induction in addition to other functions common to free-living aphids. In gall midges, a huge number of putative effector proteins are likely delivered into host tissues for host manipulation and gall induction. ${ }^{[13-16]}$ The most striking characteristic for putative gall midge effectors is a large number of highly diversified, small peptides. The most striking characteristic of $S$. chinensis saliva proteins is the high proportions of proteins with binding activities including protein-, DNA-, ATP-, and ion-binding proteins. ${ }^{[17,18]}$ No similarity has been found between $S$. chinensis saliva proteins identified here and reported gall midge salivary proteins, suggesting that each insect-plant system has a unique mechanism for gall induction.

Another interesting feature was that many of the proteins detected in the aphid saliva lacked a typical secretion signal peptide. The way for these proteins to be transported out of insect's salivary glands without a secretion signal peptide was not clear. However, similar phenomena have also been observed in other aphidplant systems, suggesting that there are likely other mechanisms to deliver proteins from salivary glands into host tissues from aphids in addition to the traditional signal peptide pathway. ${ }^{[9]}$

Here, we wanted to emphasize that this study on both collected saliva and dissected salivary glands is very preliminary due to the small amounts of samples obtained even though huge amounts of time and effort have been spent on this project. There are major challenges in both obtaining saliva and dissecting salivary glands from small insects. ${ }^{[9]}$ In this study, more than 24000 aphid individuals were used to collect saliva, and the amount of protein samples was perhaps still insufficient for a comprehensive analysis. It can be seen that much more protein species were identified from dissected salivary glands than from the collected saliva sample. This analysis may have only identified the most abundant proteins in the aphid saliva. A more effective method for saliva collection or a more sensitive technique for protein detection is needed for comprehensive analyses of tiny insects. Variation in salivary secretion has been observed on different artificial diets and at different feeding stages of aphid..$^{[11,12]}$ It is possible to establish a more efficient protocol to collect saliva from this aphid in the future. It was also very difficult to obtain large amounts of dissected salivary glands from $S$. chinensis individuals with our current dissection tools. The major challenge for gland dissection is to transfer salivary glands into RNA extraction solution after dissection. We are currently in the process to improve procedures to collect larger amounts of saliva samples and obtain more dissected salivary glands for more comprehensive analyses. Finally, $S$. chinensis has different developmental stages on two different hosts, ${ }^{[19]}$ and this study analyzed only one of the stages. Further studies on salivary proteins from other developmental stages on alternative hosts may provide more useful information on the functions of specific salivary proteins.

\section{Supporting Information}

Supporting Information is available from the Wiley Online Library or from the author.

\section{Acknowledgements}

X.C. and Z.Y. conceived experiments. Z.Y. conducted the experiments. L.M. performed the experiments. Z.Y. and L.M. analyzed data and wrote the manuscript. X.C., F.F., Y.Y., H.W., and H.C. analyzed the results. All authors reviewed the manuscript. The authors would like to acknowledge Prof. Ming-Shun Chen, Kansas State University, for his helpful comments. The LC-MS/MS analyses were processed by Mr. Shuo Chen in Beijing Proteome Research Center, China. This research is funded by the National Natural Science Foundation of China (Grant No. U1402263; 31372266; 31370651 ), and the Grant for Essential Scientific Research of Chinese National Non-profit Institute (Grant No. CAFYBB2014ZD005).

\section{Conflict of Interest}

The authors declare no conflict of interest.

\section{Keywords}

gall aphid, proteomics, saliva, salivary gland, Schlechtendalia chinensis

Received: October 11, 2017

Revised: March 14, 2018

Published online:

[1] G. X. Zhang, T. S. Zhong, Economic Insect Fauna of China, Science Press, Beijing 1983, p. 77.

[2] R. L. Blackman, V. F. Eastop, Aphids on the World's Crops: An Identification and Information Guide, Wiley \& Sons, England 2000.

[3] J. C. Carolan, D. Caragea, K. T. Reardon, N. S. Mutti, N. Dittmer, K. Pappan, F. Cui, M. Castaneto, J. Poulain, C. Dossat, D. Tagu, J. C. Reese, G. R. Reeck, T. L. Wilkinson, O. R. Edwards, J. Proteome. Res. 2011, 10, 1505.

[4] X. Tang, R. D. Frederick, J. Zhou, D. A. Halterman, Y. Jia, G. B. Martin, Science 1996, 274, 2060.

[5] J. C. Carolan, C. I. J. Fitzroy, P. D. Ashton, A. E. Douglas, T. L. Wilkinson, Proteomics 2009, 9, 2457.

[6] N. Mutti, Y. Park, J. Reese, G. Reeck, J. Insect Sci. 2006, 6, 38.

[7] S. A. K. Rao, J. C. Carolan, T. L. Wilkinson, PLoS One 2013, 8, e57413.

[8] A. B. Amira, J. Bauwens, E. De Pauw, S. Besbes, H. Attia, F. Francis, C. Blecker, J. Chem. Biol. 2017, 10, 25. 
[9] H. J. Huang, J. B. Lu, Q. Li, Y. Y. Bao, C. X. Zhang. J. Proteomics 2018, 172, 25.

[10] J. A. Vizcaino, R. G. Cote, A. Csordas, J. A. Dianes, A. Fabregat, J. M. Foster, J. Griss, E. Alpi, M. Birim, J. Contell, G. O'Kelly, A. Schoenegger, D. Ovelleiro, Y. Perez-Riverol, F. Reisinger, D. Rios, R. Wang, H. Hermjakob, Nucleic Acids Res. 2013, 41, 1063.

[11] P. W. Miles, Biol. Rev. 1999, 74, 41.

[12] J. I. B. Bos, D. Prince, M. Pitino, M. E. Maffei, J. Win, S. A. Hogenhout, PLoS Genet. 2010, 6, e 1001216.

[13] M. S. Chen, X. M. Liu, Z. Yang, H. Zhao, R. H. Shukle, J. J. Stuart, S. Hulbert, BMC Evol. Biol. 2010, 10, 296.

[14] C. Zhao, L. N. Escalante, H. Chen, T. R. Benatti, J. Qu, S. Chellapilla, R. M. Waterhouse, D. Wheeler, M. N. Andersson, R. Bao, M. Batterton,
S. K. Behura, K. P. Blankenburg, M. S. Chen, S. J. Brown, J. J. Stuart, S. Richards, Curr. Biol. 2015, 25, 613.

[15] C. Zhao, R. Shukle, L. Navarro, M. S. Chen, S. Richards, J. J. Stuart, J. Insect Physiol. 2016, 84, 22.

[16] R. Aggarval, S. Subramanyam, C. Zhao, M. S. Chen, M. O. Harris, J. J. Stuart, PLoS One 2014, 9, e100958.

[17] S. Vandermoten, N. Harmel, G. Mazzucchelli, E. D. Pauw, E. Haubruge, F. Francis, Insect Mol. Biol. 2014, 23, 67.

[18] H. J. Huang, C. W. Liu, X. H. Huang, X. Zhou, J. C. Zhuo, C. X. Zhang, Y. Y. Bao, J. Proteome Res. 2016, 15, 1883.

[19] P. Liu, Z. X. Yang, X. M. Chen, R. G. Foottit, Ann. Entomol. Soc. Am. 2014, 107, 245. 